\section{Premature Discard of Proton Pump Inhibitors: Possible Osteoporosis vs Enhanced Gastrointestinal Bleed, Adenocarcinoma Efficacy}

To the Editor: I commend Davidge Pitts and Kearns ${ }^{1}$ for alerting clinicians to the detrimental osteoporotic effects of newer pharmaceuticals that clinicians initiate but fail to reevaluate. However, I encourage clinicians to use considerable caution before accepting the authors' premature endorsement of substituting $\mathrm{H}_{2}$ receptor blockers $\left(\mathrm{H}_{2} \mathrm{RBs}\right)$ for proton pump inhibitors (PPIs). Millions of people ingest nonsteroidal anti-inflammatory drugs (NSAIDs) long term. The demographics of osteoporosis are inordinately concordant with the elderly population as well as with long-term NSAID consumption (with NSAID use related to the treatment of osteoarthritis pain, and aspirin prophylaxis for ischemic events). The thinned gastric mucosa in the elderly enhances their susceptibility to severe NSAID-induced adverse events.

Proton pump inhibitors do not simply block histamine receptor-mediated gastric parietal cell acid secretion effected by $\mathrm{H}_{2} \mathrm{RBs}$. Proton pump inhibitors terminate the final stage of acid production, and they also block parasympathetic vagus nerve-mediated acetylcholine neurotransmitter and gastrin hormone stimulation of parietal acid production, constituting a substantial improvement over $\mathrm{H}_{2} \mathrm{RBs}$. Additionally, PPIs are more efficacious than $\mathrm{H}_{2} \mathrm{RBs}$ in reducing risk of gastroduodenal ulcers, ${ }^{2}$ and, as such, they are superior in mitigating mortality risks for thousands of patients who annually experience NSAID-induced gastrointestinal bleeds (GIBs) and tens of thousands of patients who experience nonfatal hospitalization-related morbidity due to GIBs. Similarly, PPIs are superior to $\mathrm{H}_{2} \mathrm{RBs}$ in the minimization of gastroesophageal reflux symptoms, premalignant Barrett metaplasia, dysplasia, and esophageal adenocarcinoma.

Only with supraphysiologic dosing can $\mathrm{H}_{2} \mathrm{RBs}$ approximate PPI potency, but such dosing is accompanied by adverse cognitive effects in the elderly and potentiates the risk of induced falls, often a preterminal cascade event.

The fewer adverse events associated with PPIs must be considered relative to a recent prospective analysis of 161,806 postmenopausal women aged 50 to 79 years. This research identified that PPIs were not associated with hip fractures and only modestly associated with spine, forearm, wrist, or total fractures. ${ }^{3}$ The study concluded that "hip fractures were so rare" that long-term use of PPIs "made no difference" in their analysis. $^{4}$

Conflicting data regarding validity of PPI use and fracture risk must be contrasted to studies reporting that $\mathrm{H}_{2} \mathrm{RB}$ acid suppression also induces osteoporosis with hip fractures. ${ }^{5}$

Davidge Pitts and Kearns ${ }^{1}$ reported that thiazolidinediones compromise bone quality "in men concomitantly taking loop diuretics." Loop diuretics promote loss of total body calcium in the urine, whereas thiazides are calcium-sparing. Future studies assessing the merit of endorsing $\mathrm{H}_{2} \mathrm{RBs}$ substituted for

(ㄷ) 2011 Mayo Foundation for Medical Education and Research
PPIs (eg, in terms of bone loss) must control better for confounding factors such as diuretic selection. This is a prerequisite if we are to establish or refute the legitimacy of whether PPIs contribute to fracture risk.

A precipitous resumption of $\mathrm{H}_{2} \mathrm{RBs}$ with discard of the pharmacologic advancement of PPIs is currently unwarranted.

Until higher-quality evidence regarding bone and $\mathrm{H}_{2} \mathrm{RB}$ use is established relative to the use of PPIs and these outcomes are correlated with GIBs, reflux, and fall risks, clinicians should continue favoring PPIs.

However, given the evidence reported by Davidge Pitts and Kearns, cautious responsive addition of bone-enhancing strategies with calcium supplements, vitamin D, pharmaceuticals, cautious loop diuretic substitution for thiazides, and diagnostic investigation to identify contributors to impaired bone quality will optimize care for patients continuing to ingest PPIs long term.

\section{Aaron S. Geller, MD \\ Tufts School of Medicine \\ New England Medical Center Nashua, NH}

1. Davidge Pitts CJ, Kearns AE. Update on medications with adverse skeletal effects. Mayo Clin Proc. 2011;86(4):338-343.

2. Lazzaroni M, Porro GB. Management of NSAID-induced gastrointestinal toxicity: focus on proton pump inhibitors. Drugs. 2009;69(1):51-69.

3. Gray SL, LaCroix AZ, Larson J, et al. Proton pump inhibitor use, hip fracture, and change in bone mineral density in postmenopausal women: results from the Women's Health Initiative. Arch Intern Med. 2010;170(9):765-771.

4. Latimer N, Lord J, Grant RL, et al; National Institute for Health and Clinical Excellence Osteoarthritis Guideline Development Group. Cost effectiveness of COX 2 selective inhibitors and traditional NSAIDs alone or in combination with a proton pump inhibitor for people with osteoarthritis. BMJ. 2009;339:b2538.

5. Corley DA, Kubo A, Zhao W, Quesenberry C. Proton pump inhibitors and histamine-2 receptor antagonists are associated with hip fractures among atrisk patients. Gastroenterology. 2010;139(1):93-101.

doi:10.4065/mcp.2011.0237

\section{Antiretroviral Therapy and Adverse Skeletal Effects}

To the Editor: We read with interest the article by Davidge Pitts and Kearns ${ }^{1}$ entitled "Update on Medications With Adverse Skeletal Effects." In our opinion, antiretroviral compounds should also be added to the list of "bad-to-the-bone" drugs.

During the past 10 years, the average life expectancy of adults with human immunodeficiency virus (HIV) infection has increased because of effective antiretroviral therapy (ART), and the care of HIV-infected individuals has shifted from management of opportunistic infections to prevention and treatment of the metabolic complications of ART, including osteoporosis.

In particular, the synergy between HIV and/or ART-related bone damage with age-associated bone loss could lead to a serious health threat. Several clinical studies have shown that during ART, bone loss is an early event and occurs rapidly, especially within 6 months of initiation. The Strategies for Man- 
agement of Antiretroviral Therapy (SMART) study has shown that institution of an ART may be followed by a $6 \%$ decrease in bone mineral density (BMD) during the first 2 years regardless of the drug combination. Furthermore, continuous ART is associated with reduced BMD and increased fracture risk compared with intermittent, CD4 cell count-guided ART. ${ }^{2}$ Therefore, osteopenia/osteoporosis, occasionally osteomalacia, and an increased risk of fracture are reported as major adverse effects of ART, especially in a regimen including the nucleotide reverse-transcriptase inhibitor tenofovir ${ }^{3}$ and protease inhibitors.

The mechanism by which antiretroviral drugs act on the bone is multifactorial and not completely clear but is mediated in part by a direct effect on osteoblasts and osteoclasts, increased catabolism of vitamin $\mathrm{D}$, and mitochondrial damage. Interestingly, the mitochondrial damage can also cause proximal renal tubulopathy. Indeed, $1.6 \%$ to $22 \%$ of tenofovir-treated patients experience phosphate wasting and 1-hydroxylation defects of vitamin D due to proximal renal tubulopathy, leading to osteomalacia with multiple fractures, bone pain, and proximal muscle weakness.

According to the European AIDS Clinical Society guidelines, in HIV-positive patients, especially those treated with ART, dual energy x-ray absorptiometry should be performed; if BMD is abnormal, secondary causes of osteoporosis should be ruled out (eg, hypovitaminosis D, hyperparathyroidism, hyperthyroidism, malabsorption, hypogonadism/amenorrhea, autoimmune diseases, diabetes mellitus, chronic liver diseases), and lateral lumbar and thoracic spine radiography should be performed. If hypophosphatemia is present, a diagnosis of renal Fanconi syndrome should be considered.

Currently, ART-induced bone loss can be managed with a reduction of risk factors for osteoporosis, including ensuring vitamin D supplementation (800-2000 IU of vitamin $\mathrm{D}_{3}$ daily ${ }^{4}$ or up to $2000 \mathrm{IU}$ in patients treated with efavirenz ${ }^{5}$ ) to maintain a plasma 25 -hydroxyvitamin $\mathrm{D}$ concentration greater than $30 \mathrm{ng} / \mathrm{mL}(75 \mathrm{nmol} / \mathrm{L})$. Other interventions include supplemental dietary calcium (1-1.2 g/d), weight-bearing exercise, and (sometimes) bisphosphonates (oral alendronate, $70 \mathrm{mg}$ once weekly, or zoledronate, $5 \mathrm{mg}$ intravenously once yearly). Furthermore, substituting another drug for tenofovir should be considered for patients presenting with hypophosphatemia, renal Fanconi syndrome, progressive decline of glomerular filtration rate, or fragility fractures. ${ }^{4}$

We thank Ms Jacqueline Iraci for review of the English translation.

Gianluigi Fabbriciani, MD

IRCCS Humanitas Clinical Institute

Rozzano (Milano), Italy

Giuseppe V. L. De Socio, MD

Hospital "Santa Maria della Misericordia"

Perugia, Italy

Marco Massarotti, MD

IRCCS Humanitas Clinical Institute

Rozzano (Milano), Italy
1. Davidge Pitts CJ, Kearns AE. Update on medications with adverse skeletal effects. Mayo Clin Proc. 2011;86(4):338-343.

2. Grund B, Peng G, Gibert CL, et al. Continuous antiretroviral therapy decreases bone mineral density. AIDS. 2009;23:1519-1529.

3. Fux CA, Rauch A, Simcock M, et al. Tenofovir use is associated with an increase in serum alkaline phosphatase in the Swiss HIV Cohort Study. Antivir Ther. 2008;13(8):1077-1082.

4. European AIDS Clinical Society. ProMED-mail Web site. www.europeanaidsclinicalsociety.org/guidelines/g2.htm. Accessed April 2011.

5. Fabbriciani G, De Socio GV. Efavirenz and bone health [letter]. AIDS. 2009;23(9):1181.

doi:10.4065/mcp.2011.0341

In reply: We thank Geller and Fabbriciani et al for their interest in our concise review and for their thoughtful comments.

We agree with Geller that PPIs are not to be abandoned. As with all medications prescribed for an individual patient, periodic review of the ongoing need, reassessment of the riskbenefit ratio, and consideration of alternatives seem to be the rational approach. Often, PPIs are prescribed and continued without further thought or reassessment. For the appropriate patient, PPIs remain an essential therapy with an acceptable risk-benefit ratio.

Fabbriciani et al discuss another class of medications with emerging evidence of adverse skeletal effects: antiretroviral drugs used to treat HIV infections. This complex disease has wide-ranging severity and effects on the endocrine system. These medications also have wide-ranging effects that could be responsible for their negative impact on bone health. This class of medications deserves more discussion than we provided in our concise review. We agree that this class of medication warrants being added to the list of "bad-to-the-bone" drugs.

Ann E. Kearns, MD, PhD

Caroline J. Davidge Pitts, MBBCh

Mayo Clinic

Rochester, MN

doi:10.4065/mcp.2011.0280

\section{CORRECTION}

Incorrect answers for Residents' Clinic: In the Residents' Clinic entitled "35-Year-Old Woman With Recurrent Palpitations" published in the August 2011 issue of Mayo Clinic Proceedings (Mayo Clin Proc. 2011;86(8):801-804), the answers provided were incorrect. The correct answers should have read as follows: 1. e, 2. c, 3. d, 4. a, 5. e. This error occurred after final author approval.

doi:10.4065/mcp.2011.0484 